\title{
GESTIÓN DEL \\ Aseguramiento en \\ SALUd
}

Rosa Isabel Roa Buitrago*

\section{Resuman}

$\boldsymbol{E}$ l artículo describe el sistema obligatorio de aseguramiento en salud en Colombia, a cargo de las Empresas Promotoras de Salud-EPS; además realiza análisis comparativo con otros países de Latinoamérica; refleja resultados de un estudio longitudinal y descriptivo, en el que se revisan distintas investigaciones desarrolladas previamente y se recolecta información de fuentes secundarias. El aseguramiento en salud colombiano, se enmarca en la teoría de seguros y de la información, es predominantemente público y registra logros importantes en términos de cubrimiento, gastos de bolsillo, expectativas de vida saludable entre otros.

\section{Palabras clave}

Aseguramiento en salud Aseguramiento público

Gastos de bolsillo

Cubrimiento en salud

\section{Introducción}

En el presente artículo se describe la función indelegable del aseguramiento en salud, a cargo de las Empresas Promotoras de Salud-EPS del Sistema General de Seguridad Social en Salud colombiano y realiza comparación con diez países de Latinoamérica. La investigación es de tipo no experimental, de diseño longitudinal de tendencia y descriptivo; e incluye tres partes.

En la primera parte, se detalla el método utilizado. En la segunda, se presentan los resultados, se incorpora el concepto general de aseguramiento dentro del marco de la teoría de los seguros; se describe la integración del aseguramiento en el país, con el manejo

\footnotetext{
* Economista Master of Business Administration-MBA, Especialista en Evaluación Social de Proyectos de inversión; Especialista en administración en salud con énfasis en seguridad social y Magistra en Administración de Empresas.
} 
de riesgo y la calidad de los servicios, y se observan algunos resultados sobre el aseguramiento. En la tercera parte, se efectúa la discusión pertinente, con apoyo de indicadores de financiación y expectativa de vida.

\section{MÉtodo}

Ua investigación es no experimental de diseño longitudinal de tendencia y descriptiva; la unidad de análisis definida corresponde a los países seleccionados y se utilizaron fuentes de información secundaria. Para la recolec-ción de la información se diseñaron formatos en Excel.

\section{Resultados}

En Colombia, el aseguramiento en salud se encuentra enmarcado dentro de la teoría de seguros y se define (Ley 1122, 2007) como la gestión integrada y articulada (gráfica 1) del riesgo financiero, riesgo en salud, representación del afiliado ante el prestador, articulación de los servicios y garantía de la calidad en la prestación de los mismos y es a las Empresas Promotoras de Salud -EPS- a las que les corresponde realizar la gestión indelegable del aseguramiento en el país, a través de la administración de los regimenes contributivo y subsidiado.

\section{Abstract}

This article describes the Colombian Health Insurance Compulsory System in the hands of Health Promotional Companies- EPS, making a comparative analysis with other Latin American countries and giving descriptive results in which the authors review former investigations and collect information and data from secondary sources. The Colombian Health Insurance System is framed on the theory of insurance and information, being predominantly of public nature and showing significant achievements in terms of coverage, life expectation, payment, and the like.

\section{KEY WORDS}

Health insurance

Public insurance

Payment

Health coverage 
El asegurador, en este caso las EPS, protege del riesgo financiero a sus afiliados, en la eventualidad de la ocurrencia de un siniestro o contingencia por enfermedad de origen común, maternidad e incapacidad; procurando que cada familia mantenga el equilibrio financiero en su hogar (León, 2003). Las empresas promotoras a su vez, deben realizar una gestión adecuada de los recursos en el proceso de administración del riesgo financiero interno (Decreto 574,2007 ), no sólo para desarrollar la función de aseguramiento; sino tambien para permanecer en el mercado, manteniendo un capital o fondo social no inferior a 10.000 salarios mínimos legales mensuales vigentes (equivalente a $\$ 5.000$ millones), y los niveles de patrimonio, solvencia, reservas técnicas y régimen de inversiones determinados en norma legal, para evitar la perdida de capital.

GRÁFICA 1

Elementos del aseguramiento en salud

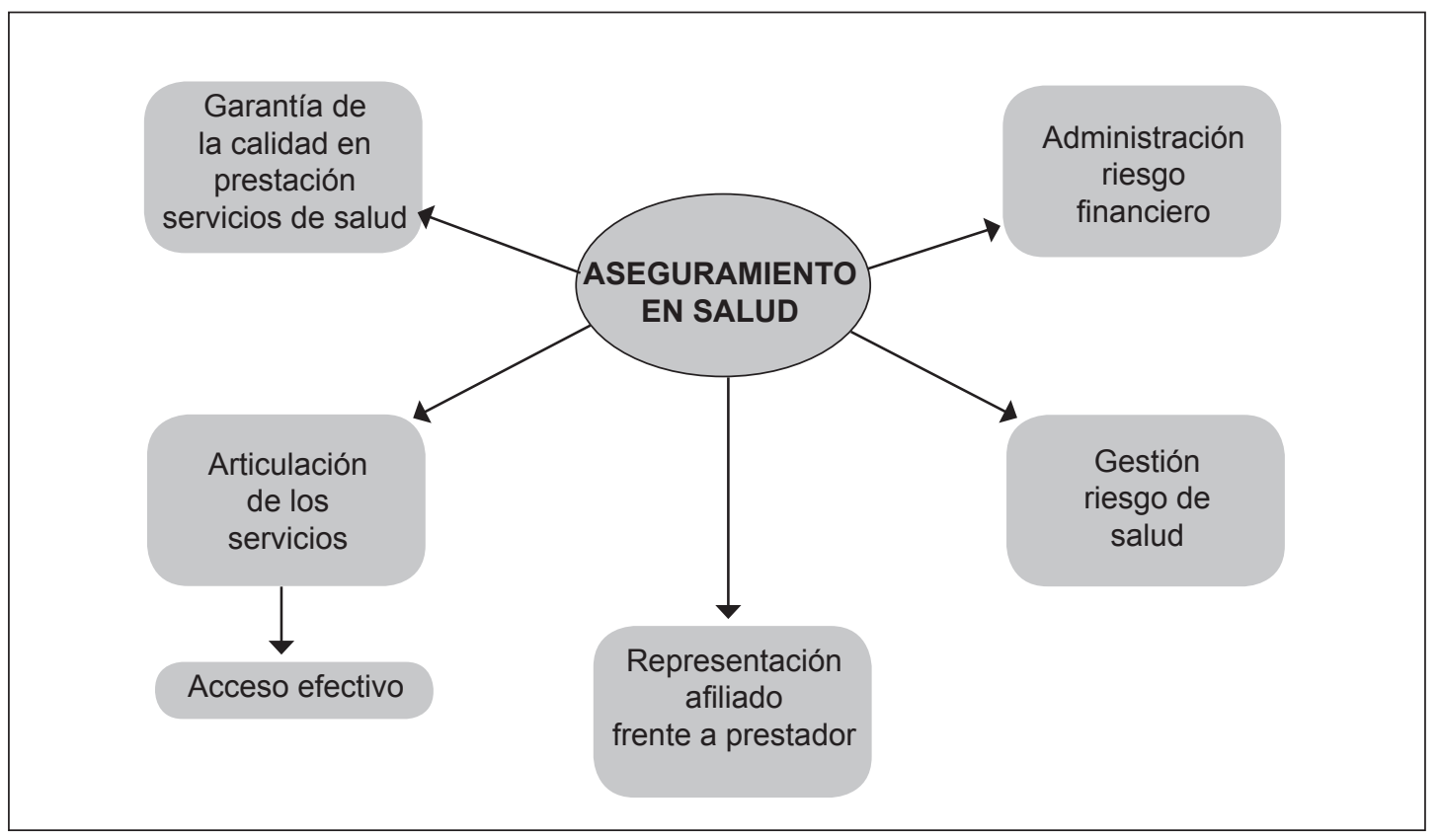

Fuente. Elaboración propia, con fundamento en los dispuesto en la Ley 1122 de 2007 "por la cual se hacen algunas modificaciones en el SGSSS y se dictan otras disposiciones". 
De otro lado, la gestión del riesgo en salud, consolidada en la Tabla 1, lo asumen las EPS, administrando el riesgo de origen común que les transfiere el afiliado y dándole la cobertura necesaria a través del Plan Obligatorio de Salud -POS-, cuando se presenta un siniestro bien sea de enfermedad, accidente, discapacidad o maternidad. Igualmente, reconoce y paga la incapacidad, licencias de maternidad $y$ paternidad, cuando es del caso.

Dada la imperfección del mercado de la salud, las aseguradoras o EPS, se enfrentan a riesgos en su gestión, que se generan por problemas de asimetría de la información, riesgo moral, selección adversa y selección del riesgo (Nicholson, 2006), que pueden generar costos adicionales.

TABLA 1

Aseguramiento público-privado sin aseguramiento Comparativo 10 paises de Latinoamérica 2006 (\%)

\begin{tabular}{|c|c|l|}
\hline Riesgo & Siniestro & \multicolumn{1}{|c|}{ Cobertura } \\
\hline & & \\
& Enfermedad & Educación \\
Accidente & Información & S \\
Común & Discapacidad & Fomento \\
& Maternidad & U \\
& & Prevención \\
& & Diagnóstico \\
& & Tratamiento \\
& & Rehabilitación \\
& & Medicamentos \\
& & Elementos \\
\hline
\end{tabular}

Fuente: elaboración propia, con fundamento en las normas legales
Para la articulación de los servicios de salud, las EPS conforman redes de Instituciones Prestadoras de Servicios de Salud -IPS-, organizadas y coordinadas con el POS, planes territoriales y planes de salud pública, cuyas acciones se orientan a la atención integral y mejoramiento del nivel de salud de la población colombiana. Este proceso genera relaciones de mercado entre aseguradores y prestadores de servicio, fundamentada en principios de competencia regulada, integración vertical patrimonial limitada y control de la posición dominante en el mercado; así como relaciones de representación de los aseguradores y los afiliados.

De otrolado, las aseguradoras o EPS, están obligadas a implementar el sistema obligatorio de garantía de la calidad en salud - SOGCS, para lo cual se cuenta con un amplio marco legal, que integra las características, componentes y normas generales orientadas fundamentalmente al seguimiento y control de las EPS y su red de servicios y por ende a mejorar en forma permanente la atención en salud centrada en el usuario (Decreto 1011, 2006); la accesibilidad a los servicios de salud, oportunidad, seguridad, pertinencia y continuidad de los mismos.

El aseguramiento en el país se financia básicamente con la Unidad de Pago de Capitación -UPC que reciben las Empresas Promotoras de Salud (EPS), previo proceso de compensación de las cotizaciones ante el Fondo de Solidaridad y Garantía -FOSYGA, 
adicionados con los recursos públicos destinados a subsidios a la demanda (Barón,2007). En otras palabras, el aseguramiento en Colombia se financia con la seguridad social, constituida a su vez con las contribuciones o aportes de los trabajadores, los empleadores (UPC-compensación) y los recursos de solidaridad.

Dado el origen de los recursos, es importante clasificar el aseguramiento. En primer lugar, el seguro público de salud de carácter general, se identifica por financiarse con recursos del gobierno, incluyendo los aportes de seguridad social, afiliación obligatoria, riesgos distribuidos entre un pool de personas de diferente perfil epidemiológico y prima fija, en el sentido de que corresponde a una contribución o aporte del salario o ingreso base de cotización; adicional a lo anterior, al seguro público tambien se le denomina seguro social, cuando este seguro es establecido por norma legal y administrado por el Gobierno; y la contribución es tripartita (empleado, empleador y aportes del presupuesto nacional). En segundo lugar, el esquema de seguro de salud privado de carácter comunitario, el cual se caracteriza por ser voluntario, ofrecer un paquete de beneficios específico, administrado por la misma comunidad a través de un pool que cubre riesgos, es simple en términos de primas y beneficios y en algunos países, el Gobierno mediante subsidios y prestación de planes de prevención, apoya la afiliación de los más pobres (Tabor, 2006). En tercer lugar, el seguro privado de salud de carácter general, el cual se administra por entidades de carácter privado y la prima usualmente está atada a la naturaleza del riesgo y a la probabilidad de ocurrencia de un evento, se financia con recursos de los particulares y puede ser de carácter obligatorio o voluntario.

Los países latinoamericanos seleccionados en el presente artículo (Argentina, Bolivia, Brasil, Chile, Ecuador, Paraguay, Perú, Uruguay y Venezuela) para ser comparados con Colombia, han reformado sus sistemas de salud usualmente bajo lineamientos de política internacional, buscando principalmente optimizar la financiación de los mismos, para lograr la universalidad, el acceso a los servicios y la equidad. Cada uno de ellos (gráfica 2), tiene sus propias características, como es el caso de Chile, Argentina y Uruguay que cuentan con sistemas de aseguramiento mixtos regulados (Madies, 2000) y una organización con diferentes subsistemas (público y privado), igual sucede con Venezuela; Paraguay a su vez, opera con tres subsistemas (público, paraestatal y privado); Brasil ha organizado un Sistema Único de Salud (SUS), en las áreas federal, estatal y municipal, con aseguramiento mixto (Acuña, 2000), financiado con impuestos; en Perú hace unos meses se aprobó la Ley Marco de Aseguramiento en Salud, la cual se encuentra en proceso de reglamentación (Chanamé, 2009) y Bolivia y Ecuador, se caracterizan por una alta segmentación y desarticulación.(OPS, 2008). 
GRÁFICA 2

Aseguramiento público-privado- sin aseguramiento

Comparativo 10 países de Latinoamérica 2006 (\%)

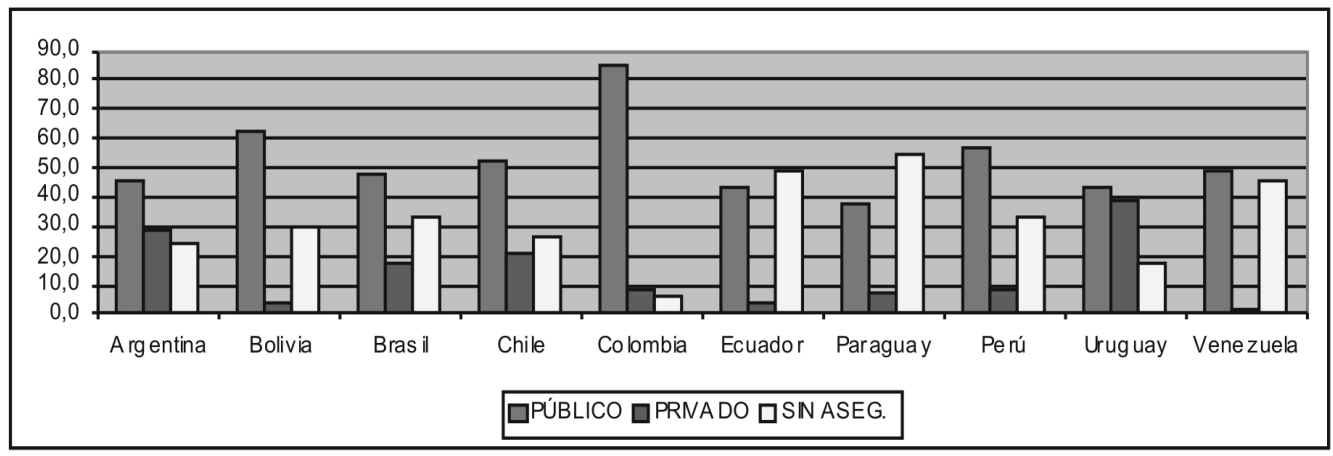

Fuente: elaboración propia, con base en WHO-World Health Statistics 2009.

\section{Discusión}

En Colombia, el aseguramiento en salud es predominantemente público, en consideración primaria a la naturaleza de los recursos con que se financia, los cuales pertenecen al Estado y en segundo lugar, por la regulación e intervención permanente del gobierno, sobre los diferentes aspectos que caracterizan la función de asegurar riesgos financieros y de salud de una población.

En otros países latinoamericanos, se han establecido sistemas de aseguramiento, predominando el seguro público en Bolivia; mixto en Argentina, Brasil, Chile, Perú y Uruguay, con igual proporción (en términos generales) de seguro público y privado adicionado con gastos de bolsillo; mientras que en Ecuador, Venezuela y Paraguay, el aseguramiento público es muy bajo, sobresaliendo el no aseguramiento, o sea el elevado gasto de bolsillo en que incurre la población para atender la prestación de sus servicios de salud.

Al comparar al país con los países latinoamericanos seleccionados, se observa que en el periodo 1996-2006, en términos generales se conserva la misma estructura de aseguramiento (gráfica 3), con excepción de Argentina país en el que disminuye el aseguramiento público y aumenta el privado; en Colombia, se presenta la situación contraria, en el sentido de que se incrementa el aseguramiento público y en Ecuador se reduce el aseguramiento público y aumenta el gasto de bolsillo o el no aseguramiento (gráfica 4). 
GRÁFICA 3

Aseguramiento público-privado- sin aseguramiento

Comparativo 10 países de Latinoamérica

Porcentaje promedio 1996-2006

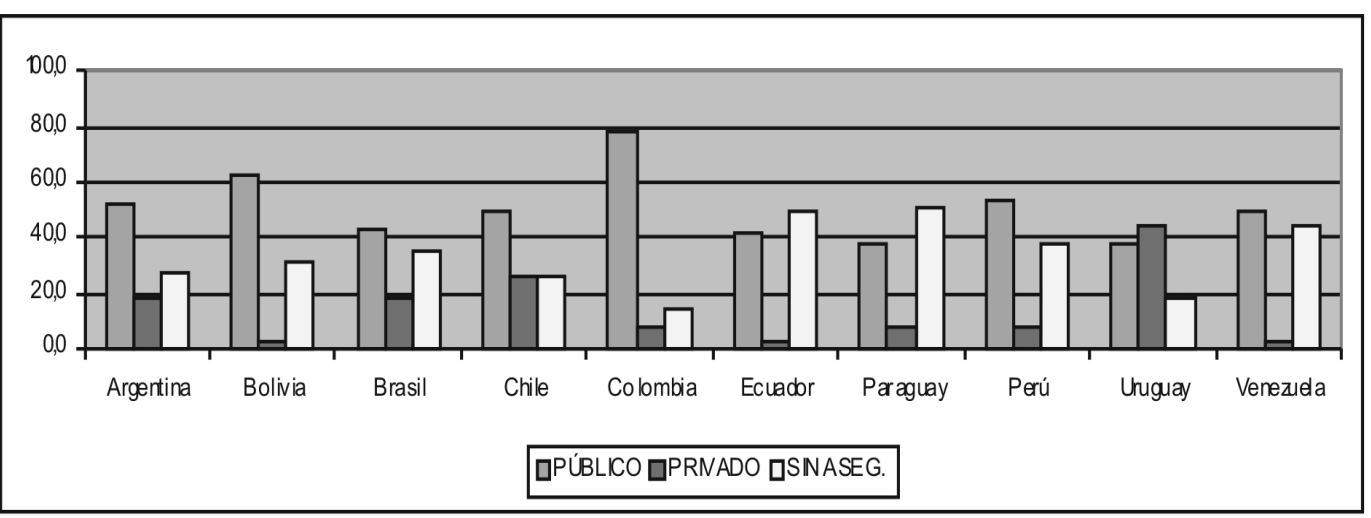

Fuente: elaboración propia, con base en WHO-World Health Statistics 2009.

GRÁFICA 4

Tendencia aseguramiento 1996-2006

Argentina-Colombia-Ecuador (\%)

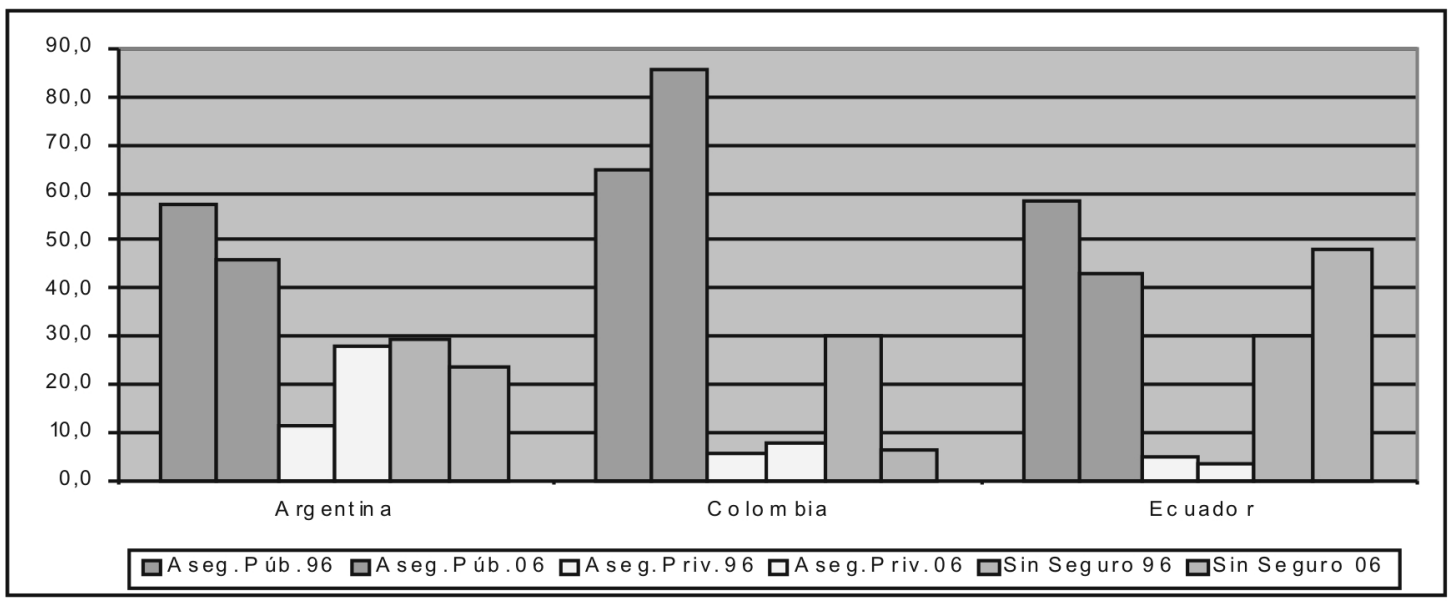

Fuente: elaboración propia, con base en WHO-World Health Statistics 2009.

El gasto de bolsillo como porcentaje del gasto total en salud, más alto se registra en Paraguay, Ecuador y Venezuela, lo que indica como se mencionó anteriormente, el desembolso que deben efectuar las personas cuando no están cubiertas por un sistema de aseguramiento general. Colombia presenta el porcentaje más bajo de gasto de bolsillo, (gráfica 5) lo que refleja mayor equidad en el aseguramiento de la población. 
GRÁFICA 5

Gasto de bolsillo comparativo 10 países latinoamericanos

Porcentaje gasto de bolsillo vs.aseguramiento 2006

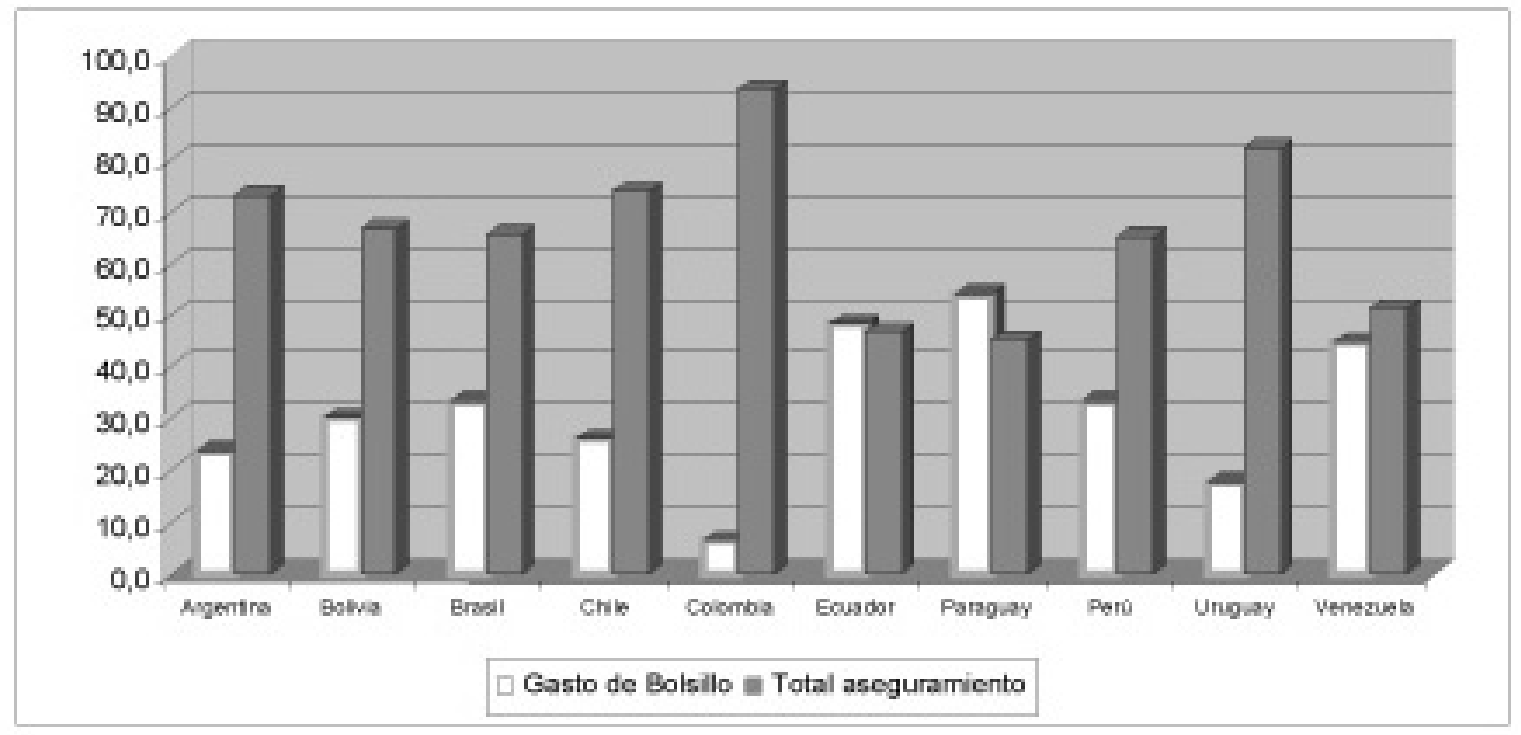

La tendencia del porcentaje del gasto de bolsillo en salud frente al gasto total en salud en Colombia, se puede observar en detalle en la gráfica
6, porcentaje que inicia en $30 \%$ en 1996 y se reduce a $6.4 \%$ en el 2006 , aspecto muy positivo en el proceso de aseguramiento.

\section{GRÁFICA 6}

Gasto de bolsilloen salud como \% del gasto total en salud en Colombia

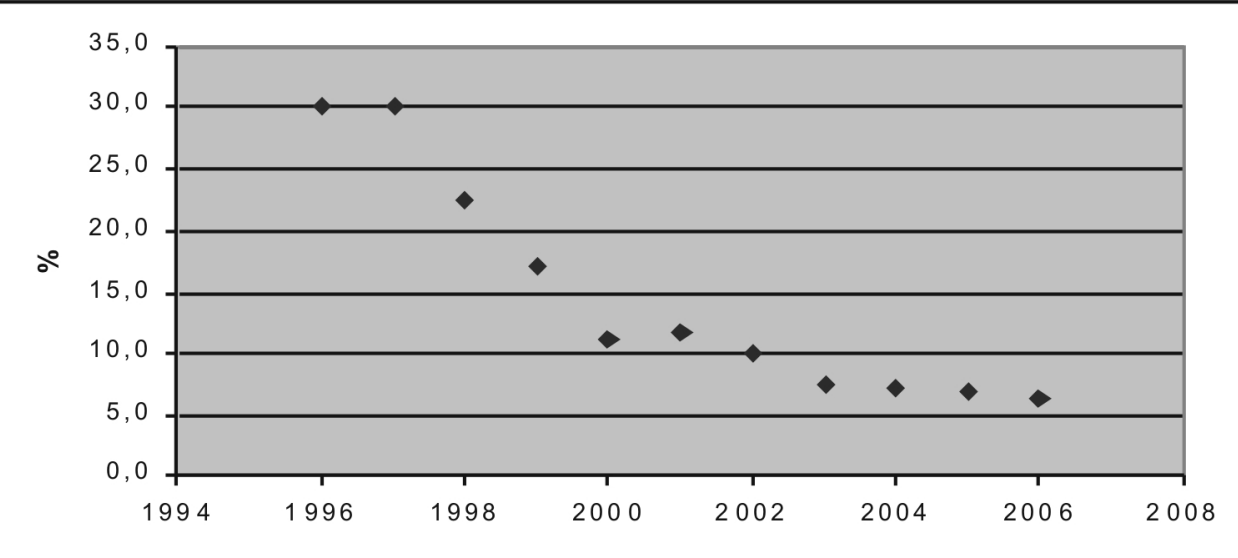

Fuente: elaboración propia. Datos extraídos de WHO-WHOSIS 
De otro lado, al evaluar los resultados comparados del aseguramiento a través de otros indicadores, se observa lo siguiente:

\subsection{Gasto per cápita en salud}

- En Colombia, en el periodo 19962006 (gráfica 7), el gasto per cápita en salud registra una tendencia irregular; es así como los años 1996-1997 dicho gasto aumenta de US\$546 a US\$614; a partir de 1998 y hasta el año 2002, la tendencia se torna decreciente, reduciéndose de US\$598 a US\$485 y en los cuatro últimos años analizados pasa de US\$499 en el 2001 a US\$626 en el 2006.

\section{GRÁFICA 7}

Gasto per cápita en salud en Colombia 1996-2006 (US\$)

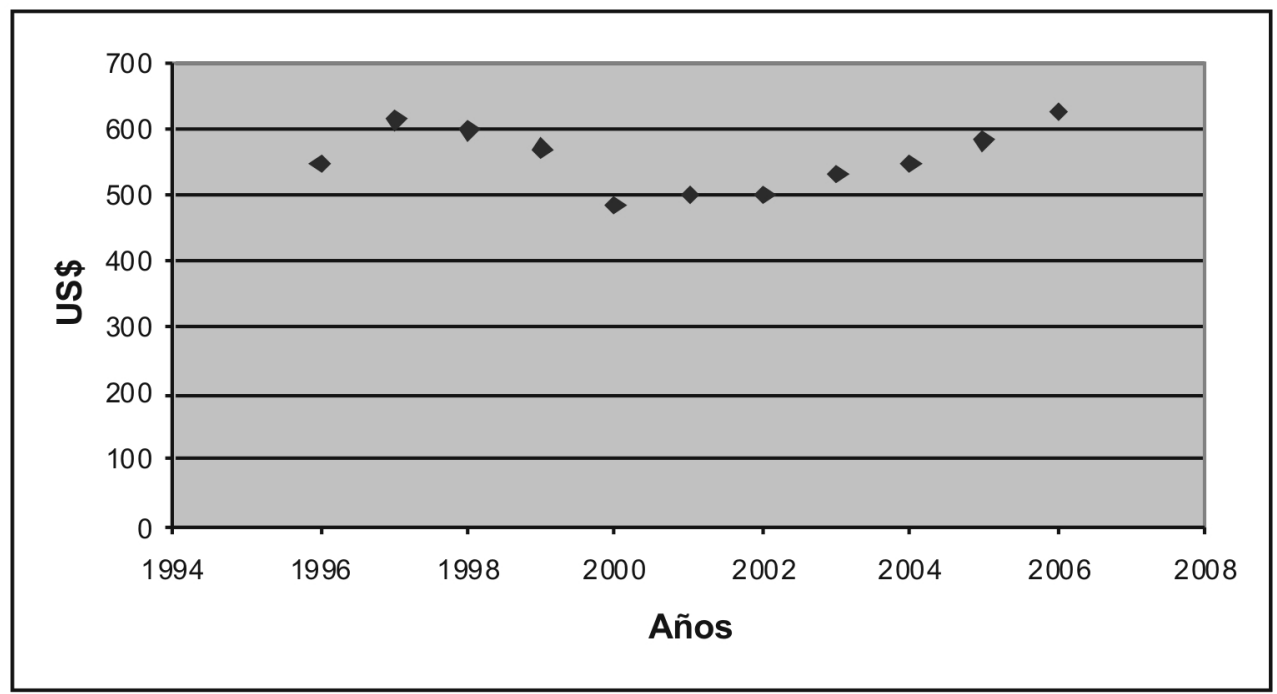

Fuente: elaboración propia. Datos extraídos de WHO-WHOSIS

- Al comparar los países (tabla 2), se observa que el gasto per cápita en salud más alto (US\$1.665) corresponde a Argentina, le siguen en su orden Uruguay (US\$989) y Brasil
(US\$765); mientras que el más bajo lo reportan Ecuador (US\$297) y Bolivia (US\$204). Colombia se encuentra en un nivel medio alto frente a los otros países (US\$626). 
TABLA 2

Gasto per cápita en salud US\$ 2006

\begin{tabular}{|l|r|}
\hline \multicolumn{1}{|c|}{ PAís } & \multicolumn{1}{c|}{ 2006 } \\
\hline Argentina & 1.665 \\
\hline Bolivia & 204 \\
\hline Brasil & 765 \\
\hline Chile & 697 \\
\hline Colombia & 626 \\
\hline Ecuador & 297 \\
\hline Paraguay & 342 \\
\hline Perú & 300 \\
\hline Uruguay & 989 \\
\hline Venezuela & 397 \\
\hline
\end{tabular}

Fuente: elaboración propia, datos extraídos de WHO - WHOSIS

- En un periodo de 10 años (gráfica 8), se observa que todos los países incrementan en algún porcentaje el gasto per capita en salud, sin embargo se destaca el significativo aumento de dicho gasto en Argentina, el cual ascendió a US $\$ 749$, el $81 \%$.

\section{GRÁFICA 8}

Gasto per cápita en salud 1996 y 2006

Comparativo 10 países de Latinoamérica (US\$)

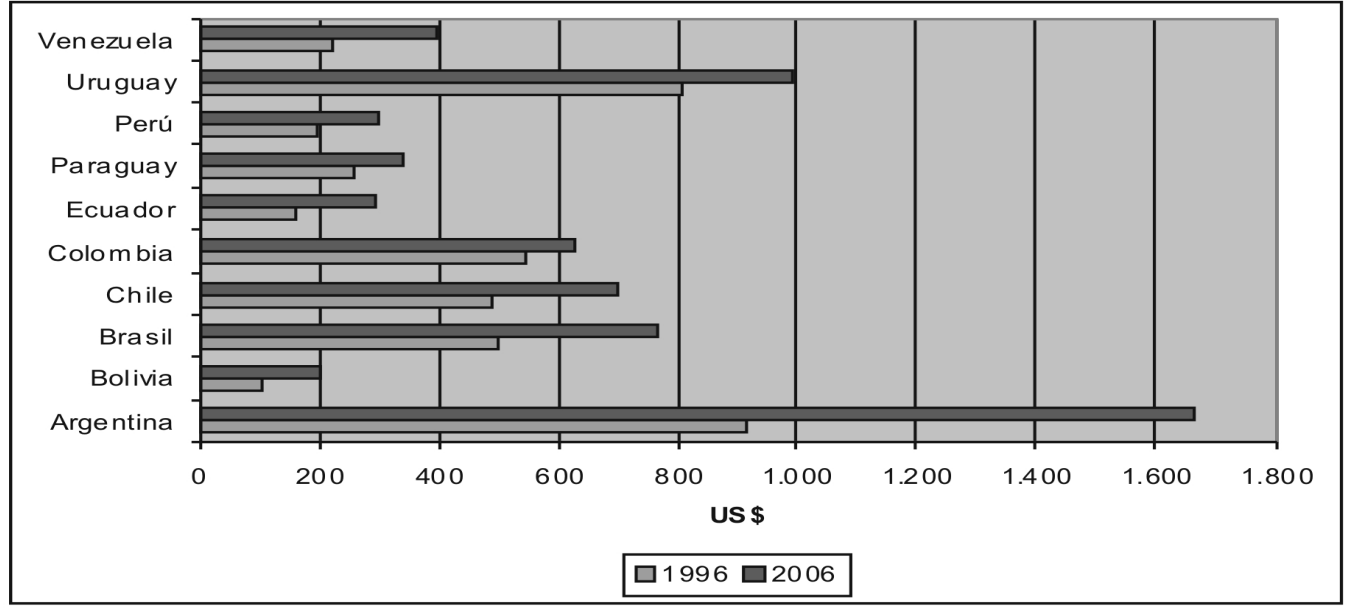

Fuente: elaboración propia. Datos extraidos de WHO-WHOSIS 


\subsection{Gasto total en salud como porcentaje del PIB}

- Este indicador (gráfica 9), demuestra la prioridad que se le da al sector salud.
- En Colombia el gasto en salud como porcentaje del PIB, aumenta en el periodo $1996-1997$ de $8.8 \%$ a $9.6 \%$, de 1998 al año 2005 decrece al pasar de $9.3 \%$ a $7.3 \%$, tendiendo a estabilizarse en $7.3 \%$ en el 2006 .

GRÁFICA 9

Gasto total en salud como \% del PIB

Colombia 1996-2006

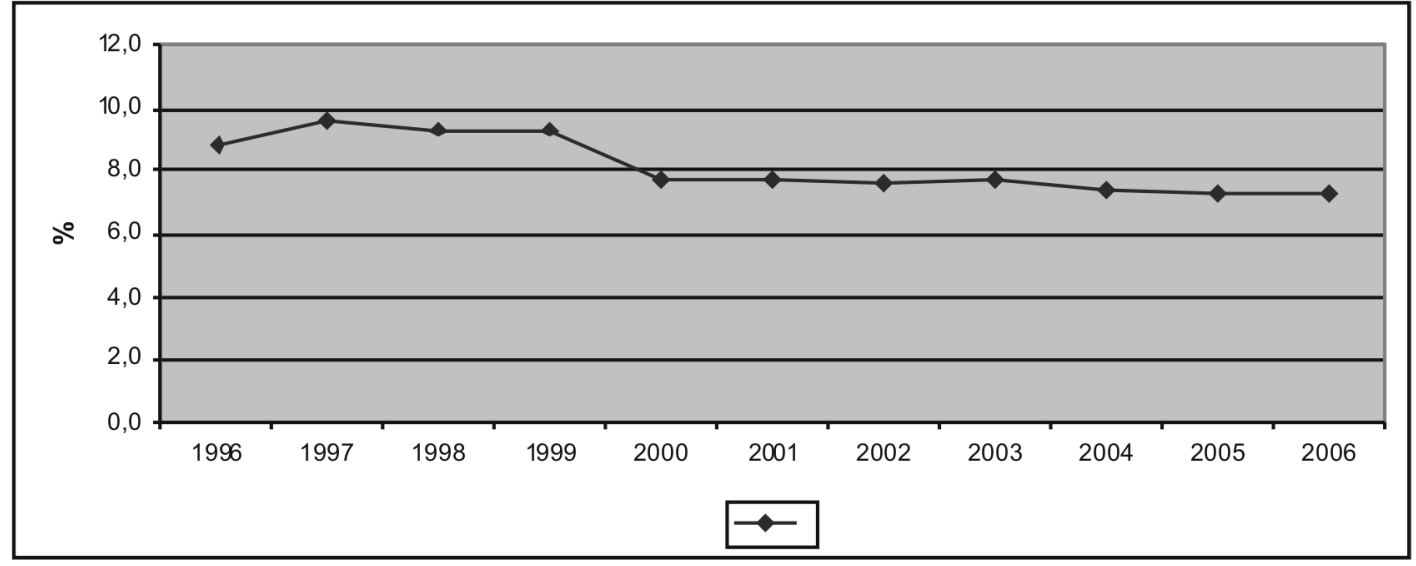

Fuente: elaboración propia. Datos extraídos de WHO-WHOSIS

De los 10 países comparados (gráfica 10), Colombia, Chile, Perú y Uruguay registran disminución en la proporción del gasto total en salud con respecto al PIB:

- De otro lado, el gasto total en salud como porcentaje del PIB (tablas 4, 5 y 6) más elevado, corresponde a Argentina (10.1\%), le siguen en su orden Uruguay (8.2\%), y Brasil (7.5\%); mientras que el más bajo lo reportan Perú y Venezuela $(4.4 \%$ y $4.9 \%$ del PIB). Colombia se encuentra en un nivel medio alto, frente a los otros países $(7.3 \%$ y US $\$ 626$, respectivamente). 
GRÁFICA 10

Gasto total como \% del PIB

Comparativo 10 países latinoamericanos

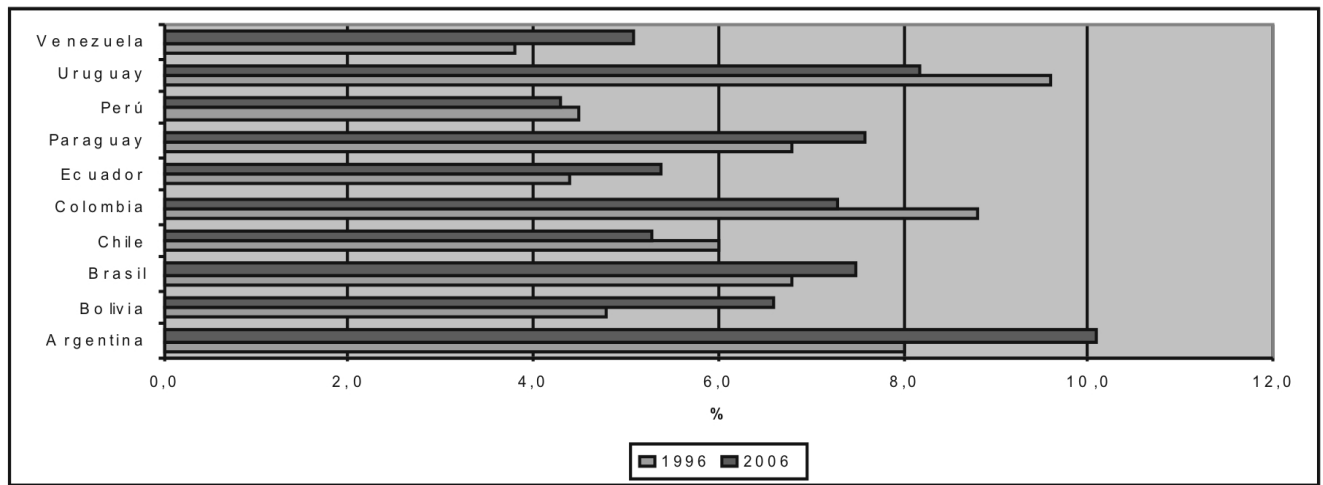

Fuente: elaboración propia. Datos extraídos de WHO-WHOSIS

\subsection{Indicadores de expectativa de vida}

Lo expuesto se complementa, observando dos importantes indicadores de mortalidad y carga de morbilidad en dichos países (gráfica 11), como son la esperanza de vida al nacer y expectativa de vida sana o saludable ${ }^{1}$.

- Las expectativas de vida de los colombianos ha mejorado en los últimos años, registrando un promedio de 74 años, correspondiendo 71 años a los hombres y 78 a las mujeres. Igualmente, la expectativa de años de vida saludables, es en el año 2003 (se han publicado datos sólo hasta el año 2003) de 62 años en promedio, destacándose el indicador para las mujeres de 66 años y mientras que el de los hombres de 58 años.

- Al comparar los países, se observa que Chile es el país que presenta los más altos indicadores de esperanza de vida al nacer (78 años) y expectativa de años saludables (70 años); mientras que Bolivia registra el más bajo (66 y 58 años, respectivamente).

\footnotetext{
${ }^{1}$ Indicador conocido con la sigla EVAS en español y HALE en inglés.
} 
GRÁFICA 11

Esperanza de vida al nacer vs. expectativa de años aludables (años)

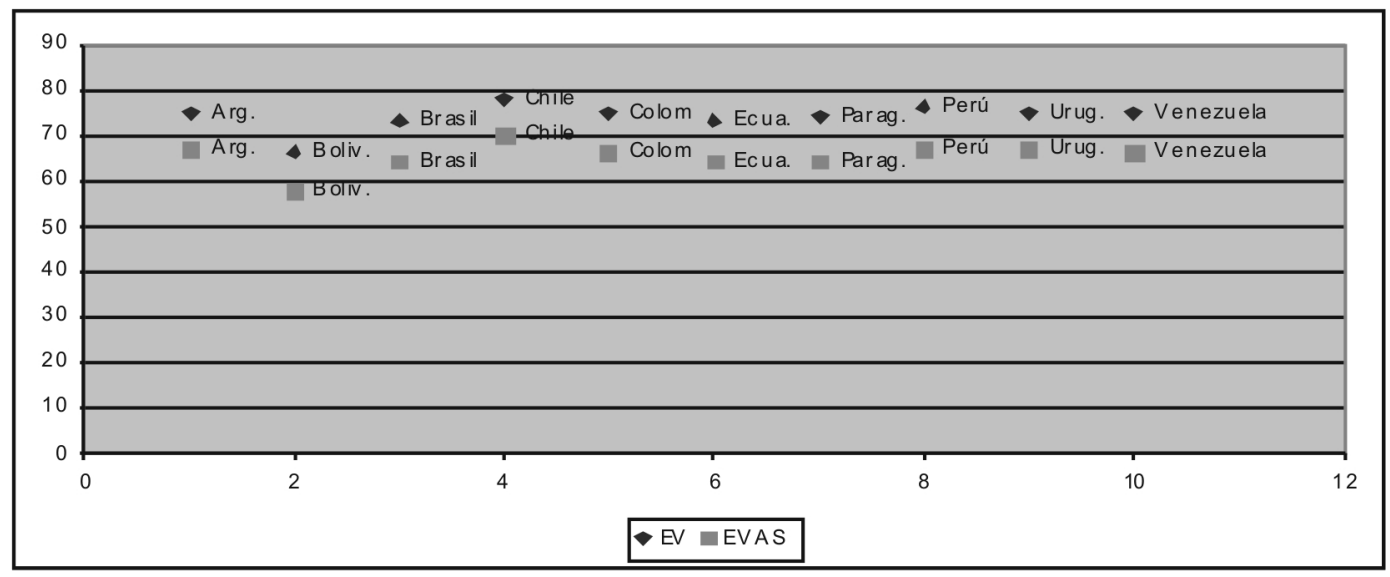

Fuente: elaboración propia. Datos extraídos de WHO-WHOSIS

Independientemente del sistema de aseguramiento que se encuentre vigente en los países comentados, los indicadores de resultado de la gestión de la salud como son el gasto de bolsillo, el gasto en salud como porcentaje del PIB, gasto per cápita en salud, esperanza de vida al nacer y expectativa de años de vida saludables, varían de un país a otro sin mostrar un patrón definido.

En el país, la estructura del aseguramiento evidentemente ha contribuido a realizar un adecuado manejo de los riesgos financieros y de salud, así como a ejercer un mayor control de los costos y gastos que se causan en la prestación del Plan Obligatorio de Salud y a que los afiliados, reciban una atención más adecuada, oportuna y de calidad. El indicador más concreto como resultado del aseguramiento en Colombia, es el número de afiliados al
SGSSS, el cual a 31 de diciembre de 2008 asciende a 39.7 millones, el 89.3\% del total, mostrando una tendencia más evidente hacia la cobertura universal, en cuanto a afiliación se refiere; aunque no hay total claridad sobre la accesibilidad a los servicios de salud por parte de toda la población asegurada.

No obstante lo anterior, la existencia de dos regimenes de salud, el contributivo y el subsidiado y un grupo de personas que no se encuentran afiliadas y clasificadas como población pobre no asegurada, conlleva a que persistan inequidades en la prestación de los servicios de salud, representadas en la existencia de dos planes obligatorios de servicios, el del régimen contributivo y el del régimen subsidiado y servicios en las Empresas Sociales del Estado para población pobre no afiliada a alguno de los regimenes. 
De otro lado, la disminución de la proporción de los gastos de bolsillo dentro del total de gastos de salud, demuestra la efectividad del aseguramiento en el país. Igualmente el estado de salud de los colombianos ha mejorado en varios frentes y el gasto per cápita en salud presenta una tendencia creciente.

En cuanto a resultados frente a otros países, Colombia se encuentra en términos generales en el punto medio en lo que respecta a gastos per cápita en salud, esperanza de vida al nacer y expectativa de años saludables de vida; se refiere, pero se destaca por ser el país que presenta el gasto de bolsillo más bajo y por ende el mayor aseguramiento en salud.

No hay un esquema definido que relacione la clase de aseguramiento que han implementado los países con los resultados esperados de salud o con lo recursos destinados para atender los servicios sanitarios, tema que podría ser desarrollado en otra investigación. 


\section{Bibliografía}

Acuña, C. (2000). Evolución y reforma de los sistemas de protección de la salud. En: los países de MERCOSUR y Chile. Extraido el 20 de mayo de 2009 de http://www.fes. org.ar/PUBLICACIONES/serie_prosur/Prosur_CeciliaAcunia.pdf

Barón L., G. Cuentas de salud de Colombia 1993 - 2006. El gasto nacional en salud y su financiamiento. Ministerio de la Protección Social - Programa de Apoyo a la Reforma de Salud - PARS, Departamento Nacional de Planeación. 2007. Extraído el 14 de octubre de 2009 de http://www.minproteccionsocial.gov.co/pars/library/ documents/DocNewsNo16237DocumentNo4262.PDF

Congreso de la República. Ley 1122. (2007). Por la cual se hacen algunas modificaciones en el Sistema General de Seguridad Social en Salud y se dictan otras disposiciones. Colombia.

León, F. (2003). De la seguridad social al aseguramiento en salud. Seminario Andino. Reformas del Sector Salud hacia el camino de la integración. "Aprendizaje y Perspectivas".

Madies, C.; Chiarvetti, S. y Chorny, M. (2000). Aseguramiento y cobertura: dos temas críticos en las reformas del sector de la salud. Revista Panamerica Salud Pública vol.8 n.1-2 Washington July/Aug. Extraído el 10 de mayo de 2009 desde http://www. scielosp.org/scielo.php?pid=S1020-49892000000700007\&script=sci_arttext

Ministerio de La Protección Social. (2009). Informe al Congreso 2008-2009. Colombia. ( $1^{\text {a }}$ ed.). Extraido el 15 de mayo de 2009 desde http://www.minproteccionsocial.gov.co

Ministerio de La Protección Social. Decreto 574 (2007). Por el cual se definen y adaptan las condiciones financieras y de solvencia del Sistema Único de Habilitación en Entidades Promotoras de Salud del Régimen Contributivo y Entidades Adaptadas. Colombia.

Nicholson, W. (2006). Teoría microeconómica. Principios básicos y ampliaciones. $\left(9^{a}\right.$ ed.). Editor Cengage Learning Editores. Extraido el 4 de junio de 2009 de http:// books.google.com.co/books?id=D-tRgvKyrikC\&pg=PA583\&dq=Walter+nicholson+ins urance $+2006 \# v=$ onepage $\& q=\& f=$ false 
OPS (2008). Perfil del sistema de salud: Ecuador, monitoreo y análisis de los procesos de cambio y reforma. Washington D.C. OPS. Extraido el 16 de mayo de 2008 de http:// www.lachealthsys.org/dmdocuments/Perfil_Sistema_Salud-Ecuador_2008.pdf

Tabor, S. (2006). Seguro de Salud Comunitario y Medidas de Protección Social. Notas sobre redes de protección social. Banco Mundial. WashingtonD.C. Extraidoel 24 de mayo de 2009, de http://siteresources.worldbank.org/SAFETYNETSANDTRANSFERS/ Resources/281945-1124119303499/SSNPrimerNote23_Sp.pdf

WHO (2009). World Health Statistics 2009. WHO 2009. Extraido el 1 de agosto de 2009 de http://www.who.org 